\title{
WHAT IS THE UNCERTAINTY IN EFFICACY OF COVID-19 VACCINES? A BAYESIAN ANALYSIS
}

December 3, 2020

\author{
Phebo D. Wibbens* \\ INSEAD, Strategy Area \\ Boulevard de Constance \\ 77305 Fontainebleau Cedex \\ France \\ Tel: +33160724000 \\ phebo.wibbens@insead.edu
}

\begin{abstract}
This short paper reports a Bayesian analysis of the publicly available COVID-19 trial results. The analysis casts some doubts on whether the half + full dose regime of the AstraZeneca COVID-19 vaccine is truly more effective than the $2 \mathrm{x}$ full dose regime. The $95 \%$ posterior interval for the efficacy of the half + full dose regime is $66.6-96.3 \%$, while for the $2 \mathrm{x}$ full dose regime it is $39.0-74.8 \%$. Hence, it is possible that in both dosage regimes the vaccine has similar efficacy, around 70\%. The estimated efficacy for the Pfizer vaccine is $89.9-97.4 \%$ and for Moderna 86.3-97.5\%. These results should be interpreted with care though, since this analysis does not account for differences in for instance trial population, COVID-19 testing, and storage requirements for the various vaccines.
\end{abstract}

*The author declares no competing interests nor any specific funding for this research. 


\section{Introduction}

Over the past weeks, data for three COVID-19 vaccines have been released. The Pfizer and Moderna vaccines exhibit around 95\% efficacy [5, 4]. The AstraZeneca vaccine had a $62 \%$ efficacy for two regular doses and a $90 \%$ when the first shot was administered in half a dose.

Though promising, these data also raise many questions. How big are the uncertainty margins for these efficacy numbers, given that these initial results are based on a limited number of COVID-19 infections? And is it really likely that a first half dose for the AstraZeneca vaccine - which was apparently administrated in error [2] - is more effective than the full dose?

A Bayesian analysis is ideally suited to address such questions. In a Bayesian analysis, the probability distribution of unknown parameters is inferred from limited known data [3]. In this case, the probability distribution of the vaccine efficacy for each trial is inferred from the publicly available data of COVID-19 infections in vaccinated and control groups.

\section{Method}

Since the infection rates as a fraction of the total trial participants is low (in the order of one or a few percent), the number of infections of the control group $n_{v}$ can be assumed to follow a Poisson distribution with rate $\lambda>0$. A vaccine with efficacy $0 \geq \alpha \geq 1$ reduces this rate to $(1-\alpha) \lambda$ for the number of infections in the vaccinated group $n_{v}$. This assumes that trial participants have equal probability of being in the vaccine or the control group. If the vaccine has no effect $(\alpha=0)$ the infection rate in the vaccinated group is the same as in the control group, and if the vaccine works perfectly $(\alpha=1)$, the rate is reduced to zero. Summarizing:

$$
\begin{aligned}
& n_{v} \sim \operatorname{Poisson}((1-\alpha) \lambda) \\
& n_{c} \sim \operatorname{Poisson}(\lambda)
\end{aligned}
$$

Assume flat priors on $\alpha$ and $\log (\lambda)$. This means $\alpha \sim \operatorname{Uniform}(0,1)$ and $p(\lambda) \propto 1 / \lambda($ an improper prior). This leads to the following posterior distribution:

$$
\begin{aligned}
p\left(\alpha, \lambda \mid n_{v}, n_{c}\right) & \propto p(\alpha) p(\lambda) p\left(n_{v} \mid \alpha, \lambda\right) p\left(n_{c} \mid \lambda\right) \\
& \propto \frac{1}{\lambda}((1-\alpha) \lambda)^{n_{v}} e^{-(1-\alpha) \lambda} \lambda^{n_{c}} e^{-\lambda} \\
& \propto(1-\alpha)^{n_{v}} \lambda^{n_{v}+n_{c}-1} e^{-(2-\alpha) \lambda}
\end{aligned}
$$

Because we are primarily interested in the distribution of the vaccine efficacy $\alpha$, the 
parameter $\lambda$ can be integrated out:

$$
\begin{aligned}
p\left(\alpha \mid n_{v}, n_{c}\right) & \propto \int_{0}^{\infty} p\left(\alpha, \lambda \mid n_{v}, n_{c}\right) d \lambda \\
& \propto \int_{0}^{\infty}(1-\alpha)^{n_{v}} \lambda^{n_{v}+n_{c}-1} e^{-(2-\alpha) \lambda} d \lambda \\
& \propto(1-\alpha)^{n_{v}} \frac{1}{(2-\alpha)^{n_{v}+n_{c}}} \int_{0}^{\infty} \lambda^{n_{v}+n_{c}-1} e^{-\lambda} d \lambda \\
& \propto \frac{(1-\alpha)^{n_{v}}}{(2-\alpha)^{n_{v}+n_{c}}}
\end{aligned}
$$

The third step in this derivation uses a parameter transformation in the integral $\lambda \mapsto \frac{\lambda}{2-\alpha}$. Since the resulting integral is constant in $\alpha$, it can be taken out of the posterior, which is commonly defined up to a normalization constant (hence the proportionality instead of equality signs). The resulting distribution is used for the posterior inference in this paper, after normalizing such that the total probability is equal to one.

The log posterior density is:

$$
\ell\left(\alpha \mid n_{v}, n_{c}\right)=\log p\left(\alpha \mid n_{v}, n_{c}\right)=n_{v} \log (1-\alpha)-\left(n_{v}+n_{c}\right) \log (2-\alpha)+c
$$

In this equation $c$ is the $\log$ of the normalization constant. The log posterior can be useful for analytical and numerical calculations. For instance, the mode of the posterior follows from $\partial \ell / \partial \alpha=0$, leading to $\alpha_{\text {mode }}=1-n_{v} / n_{c}$. This is equal to the "naïve" implied efficacy as often reported (see Table 1). Note though that the posterior median and mean are in general different from the mode - and typically more insightful. Only for large samples do these different centrality measures converge, in which case they become equivalent to maximum likelihood estimation (MLE).

\section{Data}

The vaccine developers have released only limited data so far, through press releases. Pfizer and Moderna have released the data for the number of COVID-19 cases in treatment and control groups [5, 4]. AstraZeneca states that there were 131 COVID-19 cases observed so far, while the efficacy was $90 \%$ in the half + full dose group, $62 \%$ in the $2 \mathrm{x}$ full dose group, and $70 \%$ when combining both groups [1]. This is just enough data to infer the number of COVID-19 infections in the vaccine in control groups. Table 1 shows the summary data for the different trials, including the average implied efficacy following $\bar{\alpha}=1-n_{v} / n_{c}$. 
Table 1: Trial data

\begin{tabular}{lccc}
\hline Trial & $n_{v}$ & $n_{c}$ & $\bar{\alpha}$ \\
\hline AZ-Oxford (half+full dose) & 3 & 30 & $90 \%$ \\
AZ-Oxford (2x full dose) & 27 & 71 & $62 \%$ \\
AZ-Oxford (combined) & 30 & 101 & $70 \%$ \\
Pfizer-BioNTech & 8 & 162 & $95 \%$ \\
Moderna-NIH & 5 & 90 & $94 \%$ \\
\hline
\end{tabular}

Note. Number of COVID patients in vaccine group $\left(n_{v}\right)$ and control group $\left(n_{c}\right)$ with implied average vaccine efficacy $\left(\bar{\alpha}=1-n_{v} / n_{c}\right)$, based on manufacturers' press releases of trial data $[1,5,4]$.

Figure 1: Posterior distributions
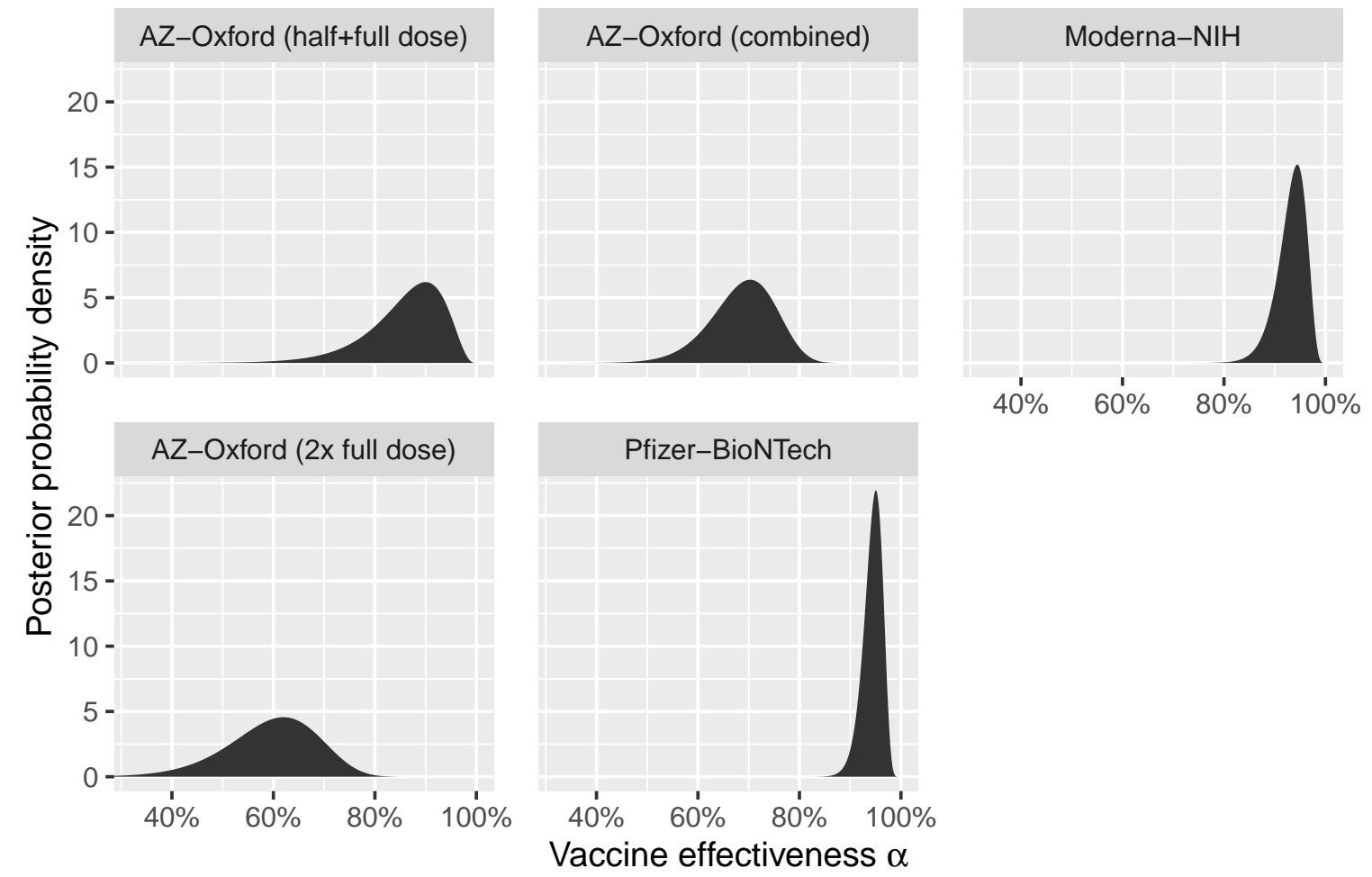

\section{Results}

The posterior distribution of $\alpha$ resulting from Equation (1) can be inferred for each trial using the data in Table 1. Figure 1 shows the resulting posterior probability density functions. Table 2 summarizes these distributions in terms of the posterior median as well as the $95 \%$ posterior probability intervals of vaccine efficacy $\alpha$.

First of all note that the posterior median (i.e., "most likely") efficacy in Table 2 is 
Table 2: Posterior inference

\begin{tabular}{lcc}
\hline Trial & Median & $95 \%$ Interval \\
\hline AZ-Oxford (half+full dose) & $87.1 \%$ & {$[66.6,96.3]$} \\
AZ-Oxford (2x full dose) & $60.2 \%$ & {$[39.0,74.8]$} \\
AZ-Oxford (combined) & $69.2 \%$ & {$[54.4,79.7]$} \\
Pfizer-BioNTech & $94.6 \%$ & {$[89.9,97.4]$} \\
Moderna-NIH & $93.6 \%$ & {$[86.3,97.5]$} \\
\hline
\end{tabular}

Note. Posterior inference of vaccine efficacy $\alpha$ expressed as a percentage.

somewhat lower than the average implied efficacy in Table 1. The reason for this can be most easily understood in the extreme case that there are no observed patients in the vaccine group, $n_{v}=0$. In such a case the implied efficacy $\bar{\alpha}$ would be $100 \%$. However, there is still a chance that the vaccine does not work perfectly, so there will be probability mass for $\alpha<100 \%$, and hence the posterior median would be below $100 \%$. A similar asymmetry is apparent in the posterior distributions in Figure 1, leading to lower posterior medians for $\alpha$ than the reported average vaccine efficacy.

Furthermore, the AstraZeneca trials exhibit wide error margins for the vaccine efficacy. Most notably, the margins are sufficiently wide that it appears conceivable that the efficacy for the half+full dose regime actually does not differ from the $2 \mathrm{x}$ full dose regime. It is possible that the vaccine has an efficacy around $70 \%$ for both dosage regimes. If the efficacy of the different dosing regimes is in fact similar, the data from these two arms of the trial could be combined. This would yield an efficacy between $54.4 \%$ and $79.7 \%$ for the AstraZeneca vaccine (95\% posterior interval).

The Pfizer and Moderna data exhibit significantly smaller error margins. The current data imply a vaccine efficacy between $89.9 \%$ and $97.4 \%$ for the Pfizer vaccine and $86.3 \%$ to $97.5 \%$ efficacy for the Moderna vaccine. These $95 \%$ intervals are strictly higher than for the AstraZeneca combined vaccine, though it still could be that the half+full dose regime of that vaccine is as effective as the Pfizer and Moderna vaccines. Clearly, more data on the AstraZeneca vaccine in the different dosage regimes would be needed to assess this.

\section{Discussion}

This study uses a simple Bayesian analysis on publicly available trial data. It comes with several limitations. Most notably, not taken into account is that vaccines and trials can differ in many ways, such as:

- Trial participant demographics (country, age, medical history, etc.)

- COVID-19 testing procedure (for instance, in the AZ-Oxford trials participants are checked 
pro-actively for asymptomatic infections, while the Pfizer and Moderna trials rely on selfreporting with follow-up tests [2])

- The storage and distribution of the vaccines (for instance, the Pfizer vaccine needs to be stored in $-70^{\circ} \mathrm{C}$, while the AstraZeneca one comes with relatively mild storage conditions, facilitating distribution, especially for less economically-developed areas)

- Other end points (such as hospitalizations) and side effects

The analysis could also be further extended. For instance, hierarchical priors could be used across different trials in order to get a sense of the distribution of vaccine efficacy. Also, a more formal Bayesian analysis could be performed to assess the posterior probability of the difference in efficacy of the two dosage regimes of the AstraZeneca vaccine. Such analysis could use earlier trial results with different dosage regimes (from COVID-19 or other vaccines) and/or expert assessments to estimate the prior probability that a half + full dose regime would be more effective than a $2 \mathrm{x}$ full dose regime. Finally, inference can be improved as more data comes available on these and other vaccines. Appendix A contains the $\mathrm{R}$ code used for the present analysis, which future researchers can use for replication of the results in this paper and for future extensions.

\section{Conclusion}

A Bayesian analysis of the publicly available trial data casts some doubt on whether the half + full dose regime of the AstraZeneca COVID-19 vaccine is truly more effective than the $2 \mathrm{x}$ full dose regime. It is possible that the vaccine has an efficacy around $70 \%$ for both dosage regimes. The data for the combined trials suggests that this vaccine might be less effective than the Pfizer and Moderna vaccines, which likely have an efficacy of at least $85 \%$ and probably above $90 \%$, up to $97.5 \%$.

\section{References}

[1] AstraZeneca. AZD1222 vaccine met primary efficacy endpoint in preventing COVID-19, November 2020.

[2] The Economist. Another covid-19 vaccine joins the party, November 2020.

[3] Andrew Gelman, John B Carlin, Hal S Stern, David B Dunson, Aki Vehtari, and Donald B Rubin. Bayesian data analysis. Chapman and Hall/CRC, 3rd edition, 2013.

[4] NIH. Promising interim results from clinical trial of NIH-Moderna COVID-19 vaccine, November 2020.

[5] Pfizer. Pfizer and BioNTech conclude phase 3 study of COVID-19 vaccine candidate, meeting all primary efficacy endpoints, November 2020. 
medRxiv preprint doi: https://doi.org/10.1101/2020.11.30.20240671; this version posted December 3, 2020. The copyright holder for this preprint (which was not certified by peer review) is the author/funder, who has granted medRxiv a license to display the preprint in perpetuity.

\section{A Appendix: $\mathrm{R}$ code}

The below $\mathrm{R}$ code generates the tables and figure presented in this paper.

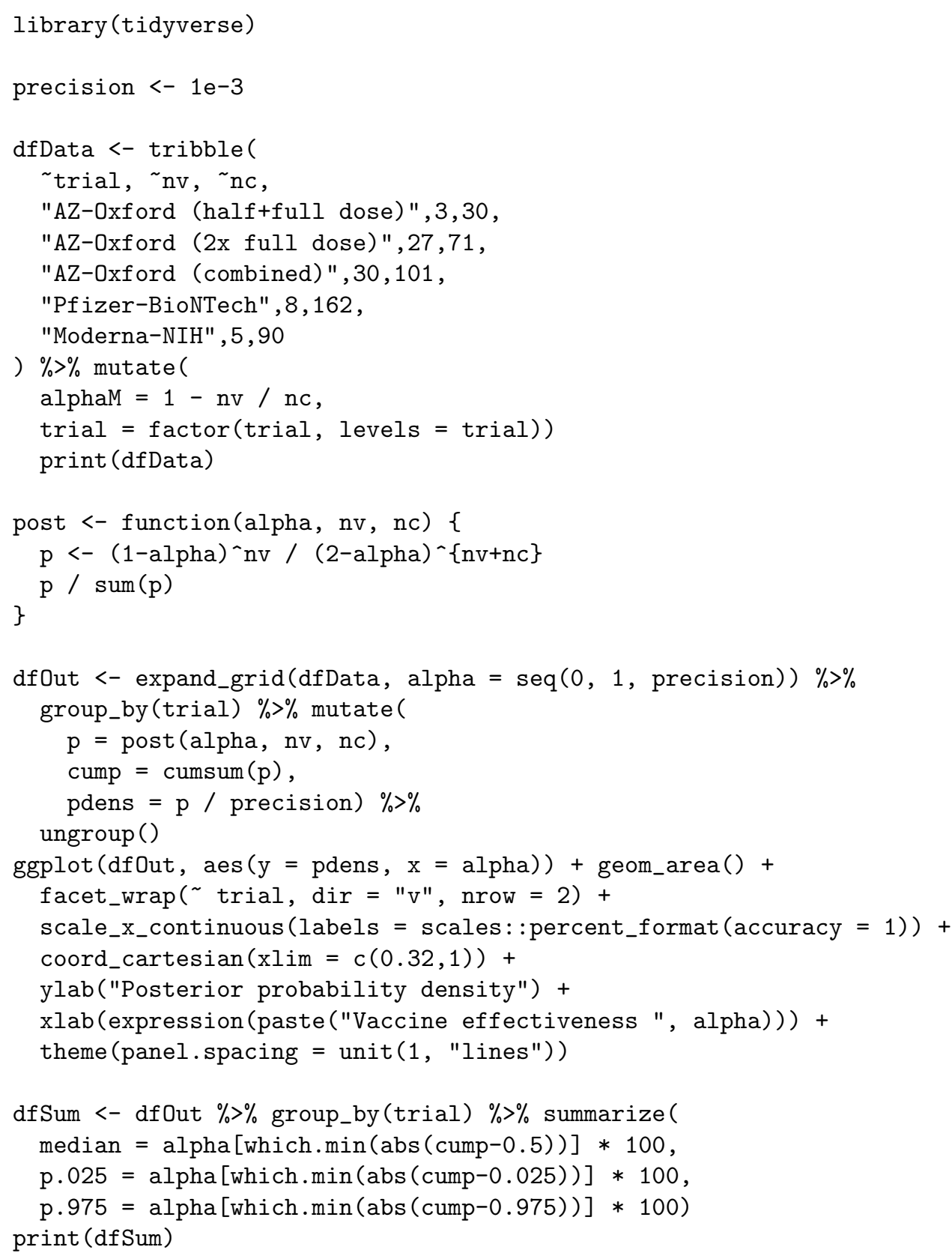

